\title{
Stage IIB Appendix Carcinoma AJCC v8
}

National Cancer Institute

\section{Source}

National Cancer Institute. Stage IIB Appendix Carcinoma A/CC v8. NCI Thesaurus. Code C134123.

Stage IIB includes: T4a, N0, M0. T4a: Tumor invades through the visceral peritoneum, including the acellular mucin or mucinous epithelium involving the serosa of the appendix or serosa of the mesoappendix. N0: No regional lymph node metastasis. M0: No distant metastasis. (AJCC 8th ed.) 